\title{
Coexistence of synchronization and anti-synchronization in chaotic systems
}

\author{
LING REN, RONGWEI GUO and UCHECHUKWU E. VINCENT
}

\begin{abstract}
The coexistence of anti-synchronization and synchronization in chaotic systems is investigated. A novel algorithm is proposed to determine the variables of the master system that should anti-synchronize with corresponding variables of the slave system. Control strategies that guarantee the coexistence of synchronization and anti-synchronization in the unified chaotic system are presented; while numerical simulations are employed to validate and illustrate the effectiveness of the proposed method.
\end{abstract}

Key words: coexistence synchronization, complete synchronization, anti-synchronization, unified chaotic system.

\section{Introduction}

Chaos synchronization was first observed by Pecora and Carroll [1] in 1990. From then on, increasing interest has been devoted to the study of chaos synchronization due to several potential applications. Recently, several typical synchronization phenomena have been identified, such as complete synchronization (CS), phase synchronization (PS), lag synchronization (LS), generalized synchronization (GS), anti-phase synchronization (AS), projective synchronization (PS), etc. A variety of works have been devoted to how to realize them (see $[2,3,4,5,6,7,8,9,10,11]$ and the references therein). It is well known that the synchronization between the master (or drive) and the slave system (or response) is equivalent to the globally asymptotically stable (GAS) of the error dynamics $e$ (the difference of the master system and slave system). Similarly, the synchronization between the master system and the slave system is equivalent to the GAS of the error dynamic system $E$ (the sum of the master system and slave system) is GAS.

L. Ren and R. Guo are with School of Science, Qilu University of Technology, Jinan 250353, China. U.E. Vincent, the corresponding author is with the Department of Physical Sciences, Redeemer's University, PMB 3005 Redemption City, Nigeria and Department of Physics, Lancaster University, Lancaster LA14YB, United Kingdom, e-mail: ue_vincent@yahoo.com

This work was supported by National Natural Science Foundation of China [61304133, 61305130, 61374074], Natural Science Foundation of Shandong Province [ZR2012AQ013, ZR2013FQ003], China Postdoctoral Science Foundation funded project [2013M541915, 2013M541912] and the Scientific Research Foundation of Shandong province Outstanding Young Scientist Award [BS2013SF023].

Received 06.10.2015. 
In order to design a simple and practically realizable controller from the control theory perspective, it is necessary that the origins of $e$ and $E$ are equilibrium points of the error systems $\dot{e}=F(y)-F(x)$, and $\dot{E}=F(y)+F(x)$, respectively, where $e=y-x$ and $E=y+x$ ). Obviously, $e=0$, i.e., $y=x$, is an equilibrium point of the error system $\dot{e}=F(y)-F(x)$. Whereas, $E=0$, i.e., $y=-x$ is an equilibrium point of the error system $\dot{E}=F(y)+F(x)$ if and only if $F(-x)=-F(x)$. Thus, realizing anti-synchronization is more complex and challenging than complete synchronization. In fact, this necessary condition is not considered in the most of the existing works on anti-synchronization of chaotic systems (See for instance Refs. [8, 9, 10, 11, 12, 13]). Moreover, the controllers obtained for achieving anti-synchronization of chaotic systems are structurally complex, i.e., some terms in those controllers are needed to counteract the redundant terms, such that $E$ is not the equilibrium point of the error system $\dot{E}=F(y)+F(x)$. For example, in Ref. [11], the $x_{2} z_{2}+x_{1} z_{1}$ term in $u_{2}$ of the equation (14) counteracts the redundant term $-x_{2} z_{2}-x_{1} z_{1}$ in the error system (13), while the $-x_{1} y_{1}-x_{2} y_{2}$ in $u_{3}$ term of the equation (14) also does same.

In addition, existing works on synchronization are mostly focus on investigating the same kind synchronization phenomenon in a given chaotic system, i.e., all the state variables of the slave (or response) system synchronize identically with the corresponding states of the master (or drive) system. For instance, if two systems achieve synchronization (or anti-synchronization, or lag-synchronization, or any other form of synchronization) with each other, it implies that each pair of the state variables between the interactive systems is completely synchronous (or anti-synchronous, or lag-synchronized, or etc). In $[14,15,16]$ and a few other works, it has been pointed out that synchronization and anti-synchronization could coexist in chaotic systems. Since there are several possible variables that could synchronize or anti-synchronize in a typical chaotic system, selecting appropriate synchronizing variables of the master to anti-synchronize the corresponding variables in slave chaotic system is of great significance in investigating the coexistence of anti-synchronization and synchronization in the same chaotic system. However, there are no results to the best of our knowledge that gives the conditions or algorithms for selecting or determining the variables of the master chaotic system that should synchronize or anti-synchronize the corresponding variables of the slave chaotic systems.

Motivated by the above discussion, we undertake a further investigation in this paper the coexistence of synchronization and anti-synchronization, in which one part of the state space of the interactive systems is in anti-phase synchronous state, while the other parts are in complete synchronous state; using the unified chaotic system as an example. Specifically, we propose here a novel algorithm which can be used to select the variables of the master system that should anti-synchronize the corresponding variables of the slave system. Then, we give some control strategies that guarantees the global asymptotic stability for the coexistence of synchronization and anti-synchronization in the unified chaotic system. Finally, the results are validated using numerical simulations. 


\section{Preliminaries}

In this section, we first give some definitions and results. Consider the following chaotic system:

$$
\dot{x}=F(x),
$$

where $x \in \mathrm{R}^{n}$ is the state, $F(x)=\left(F_{1}(x), F_{2}(x), \cdots, F_{n}(x)\right)^{T}$ is a continuous vector function, and $F(0)=0$.

Let system (1) be the master system. The corresponding slave system is given as

$$
\dot{y}=F(y)+u,
$$

where $y \in \mathrm{R}^{n}$ is the state variable and $u$ is the controller to be designed.

First, let the synchronization error state be defined as $e=y-x$, so that the error dynamics is given as follows

$$
\dot{e}=F(y)-F(x)+u,
$$

where $e \in \mathrm{R}^{n}$ is the error state space variable.

Definition 1 The master system (1) synchronizes the slave system (2) if and only if the following condition

$$
\lim _{t \rightarrow \infty}\|e(t)\|=0
$$

is satisfied.

Secondly, let the anti-synchronization error be defined by $E=y+x$, so that the error dynamics is given as follows

$$
\dot{E}=F(y)+F(x)+u,
$$

where $E \in \mathrm{R}^{n}$ is the error state variable and $u$ is the controller to be designed.

Definition 2 The master system (1) anti-synchronizes the slave system (2) if and only if the following condition

$$
\lim _{t \rightarrow \infty}\|E(t)\|=0
$$

is satisfied.

In the following, we present an algorithm to determine variables of the master system that should anti-synchronize the corresponding variables of slave system.

\section{Algorithm 1.}

Step 1: Without loss of generality, we first select the variable $x_{1}$. If $F_{1}(x)=$ $F_{11}\left(x_{1}\right)+F_{12}\left(x_{2}, \cdots, x_{n}\right)$ is an odd function, or $F_{11}\left(x_{1}\right)=\alpha_{1} x_{1}$, we can set $E_{1}=x_{1}+y_{1}$, where $\alpha_{1}$ is a real number. 
Step 2: If $F_{12}\left(x_{2}, \cdots, x_{n}\right)=\alpha_{2} x_{2}+F_{13}\left(x_{3}, \cdots, x_{n}\right)$, we should set $E_{2}=x_{2}+y_{2}$, where $\alpha_{2}$ is a real number. Else, if $F_{2}(x)=F_{21}\left(x_{2}\right)+F_{22}\left(x_{1}, x_{3}, \cdots, x_{n}\right)$ is an odd function, or $F_{21}\left(x_{2}\right)=\alpha_{3} x_{2}$, we can set $E_{2}=x_{2}+y_{2}$, where $\alpha_{3}$ is a real number. Then, we determine whether $E_{2}=x_{2}+y_{2}$ is suitable or not according to the condition that origin is an equilibrium point of the error system.

Step 3: When $i \leqslant n$, we can set $E_{i}=y_{i}+x_{i}$ or $e_{i}=y_{i}-x_{i}$ by the similar procedure in Step 2.

Remark 1 If $F(x)$ is an odd vector function, we can simultaneously synchronize and anti-synchronize two identical chaotic or hyper-chaotic systems using same controller. In Ref. [15], we have simultaneously synchronized and anti-synchronized two identical new 4D chaotic systems.

\section{Main results}

In this section, we use the unified chaotic system as an example of a typical chaotic systems to illustrate how the coexistence of synchronization and anti-synchronization in chaotic systems can be achieved using the algorithm described above. According to $[17,18,19,20]$, the unified chaotic system is given as

$$
\begin{aligned}
\dot{x}_{1} & =(25 \theta+10)\left(x_{2}-x_{1}\right) \\
\dot{x}_{2} & =(28-35 \theta) x_{1}+(29 \theta-1) x_{2}-x_{1} x_{3} \\
\dot{x}_{3} & =x_{1} x_{2}-\frac{8+\theta}{3} x_{3}
\end{aligned}
$$

where $\theta \in[0,1]$. System (5) is called the general Lorenz system when $\theta \in[0,0.8)$. When $\theta \in(0.8,1]$, system (5) is a general Chen system, while the Lü system is a special case of system (5) with $\theta=0.8$.

According to Algorithm 1, we can set $E_{1}=y_{1}+x_{1}$ since $f_{1}(x)=(25 \theta+10)\left(x_{2}-x_{1}\right)$, and then we should set $E_{2}=y_{2}+x_{2}$. If we set $E_{3}=y_{3}+x_{3}$, then

$$
\begin{aligned}
\dot{E}_{3} & =-\frac{8+\theta}{3} E_{3}+x_{1} x_{2}+y_{1} y_{2} \\
& =-\frac{8+\theta}{3} E_{3}+x_{1} x_{2}+\left(E_{1}-x_{1}\right)\left(E_{2}-x_{2}\right) \\
& =-\frac{8+\theta}{3} E_{3}+E_{1} E_{2}-x_{1} E_{2}-x_{2} E_{1}+2 x_{1} x_{2}
\end{aligned}
$$

It is clear that $E=0$ is not an equilibrium point of the error system (6). In fact, the left hand side of the error system (6) equals zero. However, the right hand side of the error system (6) is not equals zero, but equals $2 x_{1} x_{2}$. Therefore, we should set $e_{3}=y_{3}-x_{3}$, and obtain the error system given as (8). 
Next, let system (5) be the master system, the corresponding slave system without controller is as follows

$$
\begin{aligned}
& \dot{y}_{1}=(25 \theta+10)\left(y_{2}-y_{1}\right) \\
& \dot{y}_{2}=(28-35 \theta) y_{1}+(29 \theta-1) y_{2}-y_{1} y_{3} \\
& \dot{y}_{3}=y_{1} y_{2}-\frac{8+\theta}{3} y_{3}
\end{aligned}
$$

Then, let $E_{i}=y_{i}+x_{i}(i=1,2)$, and $e_{3}=y_{3}-x_{3}$, so that the error system is obtained as

$$
\begin{aligned}
& \dot{E}_{1}=f_{1}\left(x, y, e_{3}, E\right)=(25 \theta+10)\left(E_{2}-E_{1}\right) \\
& \dot{E}_{2}=f_{2}\left(x, y, e_{3}, E\right)=(28-35 \theta) E_{1}-E_{1} e_{3}-x_{3} E_{1}+x_{1} e_{3}+(29 \theta-1) E_{2} \\
& \dot{e}_{3}=f_{3}\left(x, y, e_{3}, E\right)=E_{1} E_{2}-x_{2} E_{1}-x_{1} E_{2}-\frac{8+\theta}{3} e_{3}
\end{aligned}
$$

where $E=\left(E_{1}, E_{2}\right)^{T}$.

Remark 2 Since system (5) is chaotic, for the uncontrolled error system (8), there exist $\lambda_{i}>0(i=1,2,3)$, which satisfies

$$
E_{i} f_{i}\left(x, y, e_{3}, E\right) \leqslant \lambda_{i} E_{i}^{2}, i=1,2, e_{3} f_{3}\left(x, y, e_{3}, E\right) \leqslant \lambda_{3} e_{3}^{2} .
$$

Note that if $E_{2}=0$, the following subsystem of system (8)

$$
\begin{aligned}
& \dot{E}_{1}=-(25 \theta+10) E_{1} \\
& \dot{e}_{3}=-x_{2} E_{1}-\frac{8+\theta}{3} e_{3}
\end{aligned}
$$

is GAS regardless of the value of $\theta$.

Therefore, we can add the controller $u=\left(0, k_{1} E_{2}, 0\right)^{T}$ to the slave system ( 7$)$ and the controlled error system the becomes as

$$
\begin{aligned}
& \dot{E}_{1}=(25 \theta+10)\left(E_{2}-E_{1}\right), \\
& \dot{E}_{2}=(28-35 \theta) E_{1}-E_{1} e_{3}-x_{3} E_{1}+x_{1} e_{3}+(29 \theta-1) E_{2}+k_{1} E_{2}, \\
& \dot{e}_{3}=E_{1} E_{2}-x_{2} E_{1}-x_{1} E_{2}-\frac{8+\theta}{3} e_{3},
\end{aligned}
$$

where the feedback gain $k_{1}$ is adapted according to the following update law

$$
\dot{k}_{1}=-\gamma E_{2}^{2},
$$

and $\gamma>0$ is an arbitrary number.

Next, we shall prove that the coexistence of synchronization and antisynchronization in the unified chaotic systems is realized by the above controller $u=$ $\left(0, k_{1} E_{2}, 0\right)^{T}$, where $x_{1}, x_{2}$ anti-synchronizes $y_{1}, y_{2}$; while $x_{3}$ synchronizes $y_{3}$. 
In order to facilitate the analysis, we refer to system (11) and (12) as the augment systems, and introduce the following positive definite Lyapunov function

$$
V\left(E, e_{3}, k_{1}\right)=\frac{1}{2}\left(e_{3}^{2}+E^{T} E\right)+\frac{1}{2 \gamma}\left(k_{1}+L_{1}\right)^{2}
$$

where

$$
L_{1}>M \sup _{E_{2} \neq 0} \frac{e_{3}^{2}+E^{T} E}{E_{2}^{2}}, \quad M=\max _{i=1}^{3} \lambda_{i} .
$$

Then, we give the following main result.

Theorem 13 Starting from any initial values of the controlled error system (11), the orbits $(E(t), e(t))^{T}$ converge to origin as $t \rightarrow \infty$, i.e., the coexistence of synchronization and anti-synchronization in the unified chaotic systems is realized by the controller $u=\left(0, k_{1} E_{2}, 0\right)^{T}$.

Proof Differentiating the Lyapunov function $V$ along the trajectories of the augment system, we obtain

$$
\begin{aligned}
\dot{V} & =E_{1} \dot{E}_{1}+E_{2} \dot{E}_{2}+e_{3} \dot{e}_{3}+\frac{1}{\gamma}\left(k_{1}+L_{1}\right) \dot{k}_{1} \\
& =E_{1} f_{1}\left(x, y, e_{3}, E\right)+E_{2}\left(f_{2}\left(x, y, e_{3}, E\right)+k_{1} E_{2}\right)+e_{3} f_{3}\left(x, y, e_{3}, E\right)-\left(k_{1}+L_{1}\right) E_{2}^{2} \\
& =E_{1} f_{1}\left(x, y, e_{3}, E\right)+E_{2} f_{2}\left(x, y, e_{3}, E\right)+e_{3} f_{3}\left(x, y, e_{3}, E\right)-L_{1} E_{2}^{2} \\
& \leqslant M\left(E_{1}^{2}+E_{2}^{2}+e_{3}^{2}\right)-L_{1} E_{2}^{2}<0,
\end{aligned}
$$

According to the Lyapunov stability theory, the equilibrium point of the error system (8) is GAS. This completes the proof.

To validate the above theoretical results, we carry out numerical simulations with the following choice of initial conditions: $x_{1}(0)=-2, x_{2}(0)=3, x_{3}(0)=4, y_{1}(0)=$ $4, y_{2}(0)=-1, y_{3}(0)=-2, \theta=0.75$ and $k_{1}(0)=-1$. Figure 1 shows the dynamics of the error systems $\left(E_{1}, E_{2}, e_{3}\right)$, illustrating global stabilization, which implies that the coexistence of synchronization and anti-synchronization in the unified chaotic systems is realized by the above controller. In Figure 2, the state variables are plotted. Clearly, we find that that $x_{1}, x_{2}$ anti-synchronizes $y_{1}, y_{2}$ while $x_{3}$ synchronizes $y_{3}$, respectively.

Next, we illustrate the coexistence of synchronization and anti-synchronization in the unified chaotic system when $\theta=0$. Proceeding as before, let system (5) be the master system, and the corresponding slave system without controller given as (7). Let $E_{i}=$ $y_{i}+x_{i}(i=1,2), e_{3}=y_{3}-x_{3}$, the obtained error system is as follows

$$
\dot{E}_{1}=10\left(E_{2}-E_{1}\right)
$$




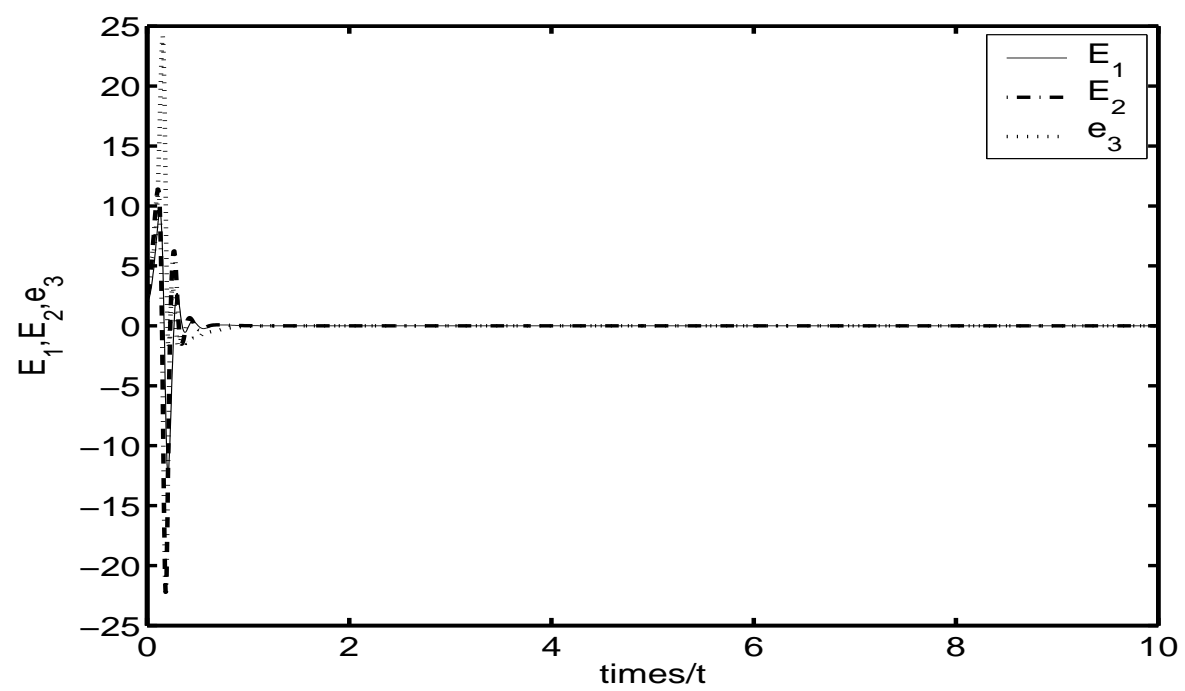

Figure 1. Error dynamics when the controller has been activated, showing that $\left(E_{1}, E_{2}, e_{3}\right)$ are stabilized, implying that the coexistence of synchronization and anti-synchronization in the unified chaotic system is realized. $\theta=0.75$ and $k_{1}(0)=-1$, while the initial conditions are $x_{1}(0)=-2, x_{2}(0)=3, x_{3}(0)=4$, $y_{1}(0)=4, y_{2}(0)=-1, y_{3}(0)=-2$.
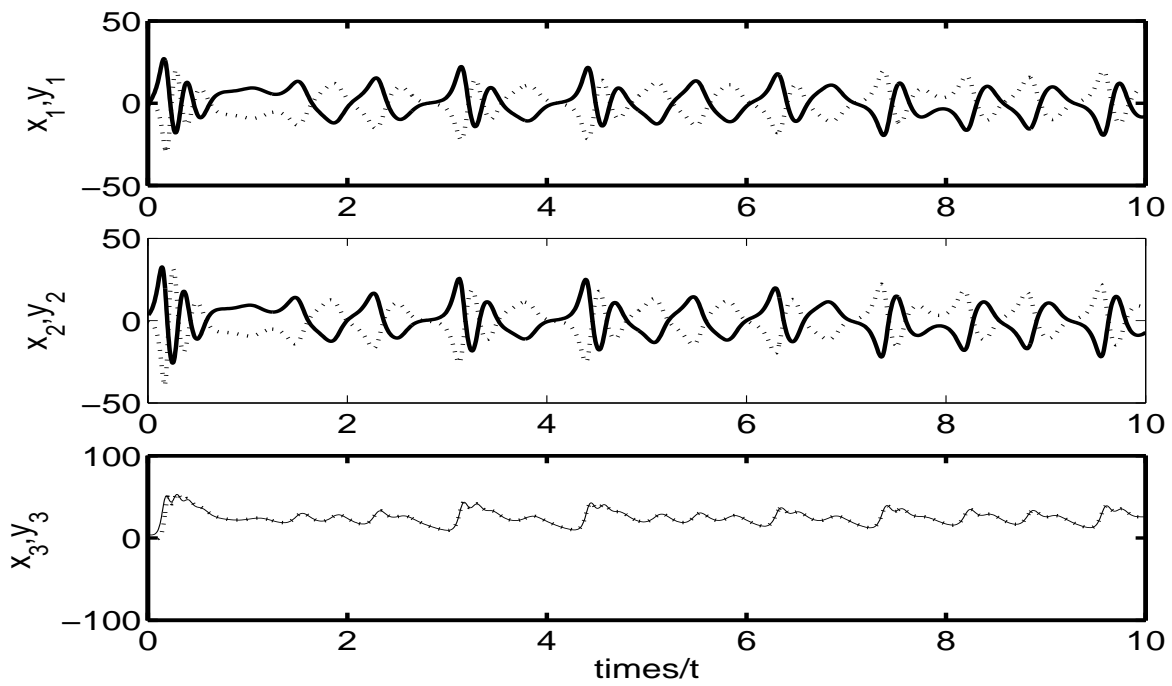

Figure 2. Dynamics of the state variables when controller has been activated. $x_{1}, x_{2}$ anti-synchronizes $y_{1}, y_{2}$ while $x_{3}$ synchronizes $y_{3} . \theta=0.75$ and $k_{1}(0)=-1$, while the initial conditions are $x_{1}(0)=-2, x_{2}(0)=$ $3, x_{3}(0)=4, y_{1}(0)=4, y_{2}(0)=-1, y_{3}(0)=-2$. 


$$
\begin{aligned}
& \dot{E}_{2}=28 E_{1}-E_{1} e_{3}-x_{3} E_{1}+x_{1} e_{3}-E_{2} \\
& \dot{e}_{3}=E_{1} E_{2}-x_{2} E_{1}-x_{1} E_{2}-\frac{8}{3} e_{3}
\end{aligned}
$$

Obviously, if $E_{1}=0$, the following subsystem of the system (15)

$$
\begin{aligned}
& \dot{E}_{2}=x_{1} e_{3}-E_{2} \\
& \dot{e}_{3}=-x_{1} E_{2}-\frac{8}{3} e_{3}
\end{aligned}
$$

is GAS.

Thus, we can add the controller $u=\left(k_{2} E_{1}, 0,0\right)^{T}$ to the slave system (7) and the controlled error system becomes

$$
\begin{aligned}
& \dot{E}_{1}=10\left(E_{2}-E_{1}\right)+k_{2} E_{1}, \\
& \dot{E}_{2}=28 E_{1}-E_{1} e_{3}-x_{3} E_{1}+x_{1} e_{3}-E_{2}, \\
& \dot{e}_{3}=E_{1} E_{2}-x_{2} E_{1}-x_{1} E_{2}-\frac{8}{3} e_{3},
\end{aligned}
$$

where the feedback gain $k_{2}$ is adapted according to the following update law

$$
\dot{k}_{2}=-\gamma E_{1}^{2}
$$

and $\gamma>0$ is an arbitrary number.

Next, we shall give the following corollary that proves that the coexistence of synchronization and anti-synchronization in the unified chaotic systems is realized by the above controller $u=\left(k_{2} E_{1}, 0,0\right)^{T}$, where $x_{1}, x_{2}$ anti-synchronizes $y_{1}, y_{2}$ while $x_{3}$ synchronizes $y_{3}$, respectively.

Corollary 1 Starting from any initial values of the controlled error system (17), the orbits $(E(t), e(t))^{T}$ converge to origin as $t \rightarrow \infty$, implying that the coexistence of synchronization and anti-synchronization in the unified chaotic systems is realized by the controller $u=\left(k_{2} E_{1}, 0,0\right)^{T}$.

Remark 3 The proof procedure is similar to the proof of Theorem 13; and therefore omitted here for brevity.

To illustrate the validity of the above result, we carry out numerical simulation result with the following choices of initial conditions: $x_{1}(0)=-2, x_{2}(0)=3, x_{3}(0)=4$, $y_{1}(0)=4, y_{2}(0)=-1, y_{3}(0)=-2$ and $k_{2}(0)=-1$. Figure 3 shows that the error system is stabilized, which implies that the coexistence of synchronization and antisynchronization in the unified chaotic systems is realized by the above controller. Furthermore, in Figure 4 the state variables are plotted; in which it is clearly shown that $x_{1}, x_{2}$ anti-synchronizes $y_{1}, y_{2}$ while $x_{3}$ synchronizes $y_{3}$. 


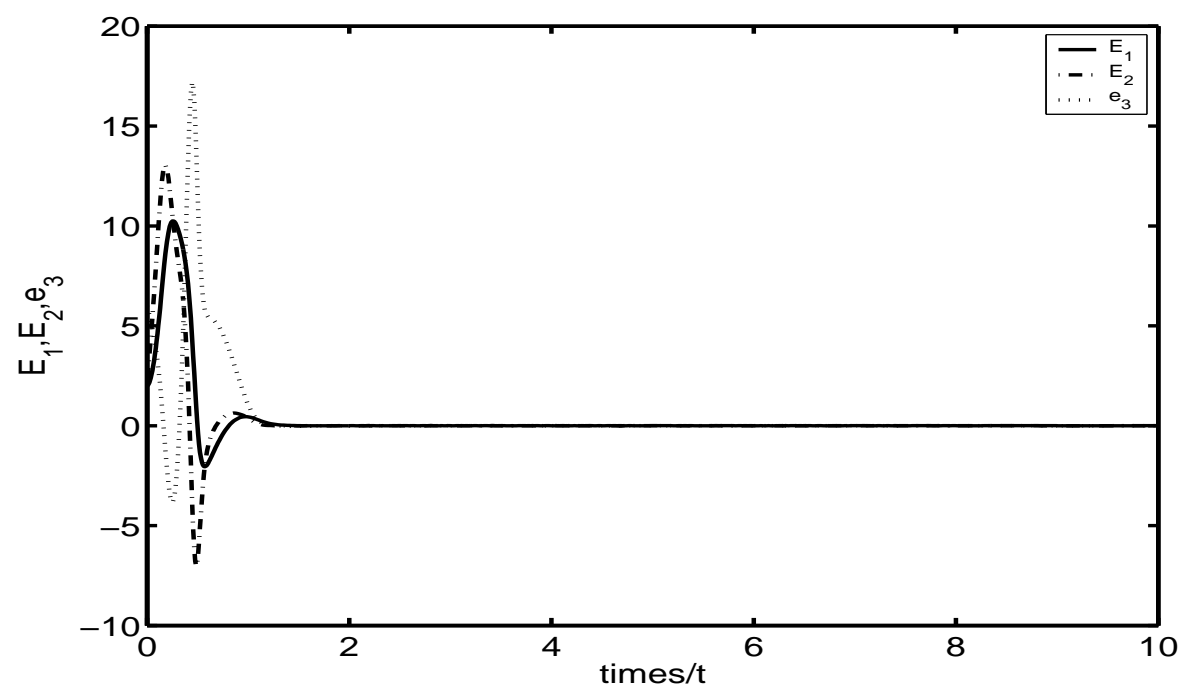

Figure 3. Error dynamics when the controller has been activated, showing that $\left(E_{1}, E_{2}, e_{3}\right)$ are stabilized, implying that the coexistence of synchronization and anti-synchronization in the unified chaotic system is realized. $\theta=0$ and $k_{2}(0)=-1$, while the initial conditions are $x_{1}(0)=-2, x_{2}(0)=3, x_{3}(0)=4, y_{1}(0)=$ $4, y_{2}(0)=-1, y_{3}(0)=-2$.
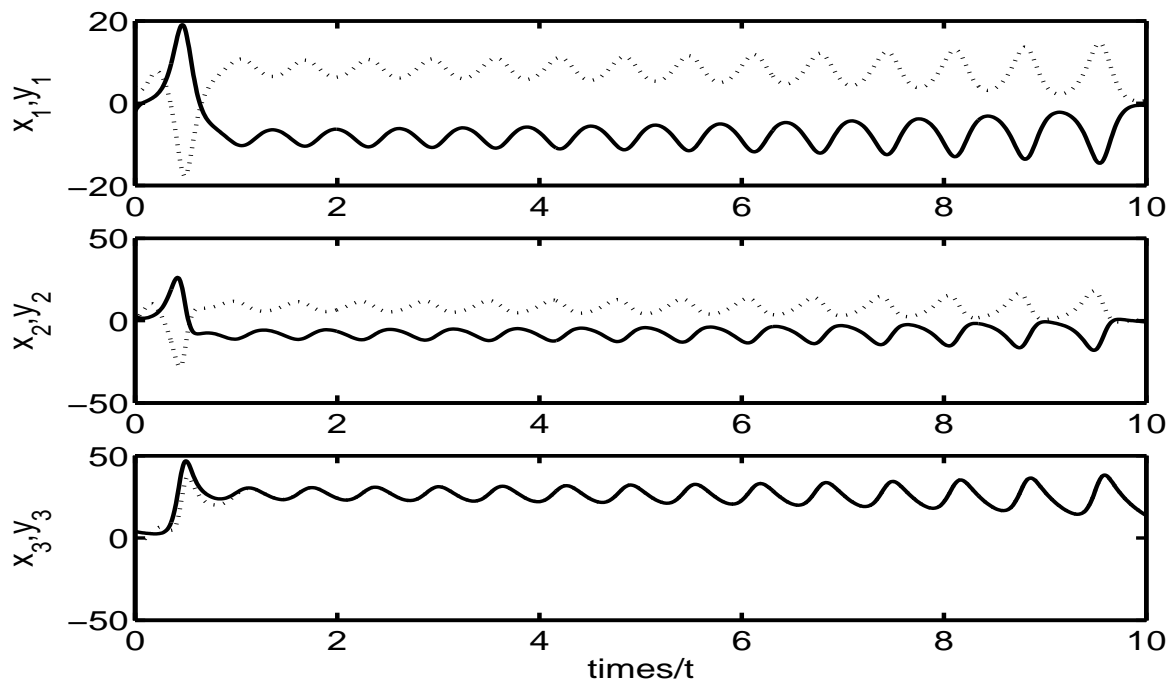

Figure 4. Dynamics of the state variables when controller has been activated. $x_{1}, x_{2}$ anti-synchronizes $y_{1}, y_{2}$ while $x_{3}$ synchronizes $y_{3} . \theta=0$ and $k_{2}(0)=-1$, while the initial conditions are $x_{1}(0)=-2, x_{2}(0)=$ $3, x_{3}(0)=4, y_{1}(0)=4, y_{2}(0)=-1, y_{3}(0)=-2$. 


\section{Conclusion}

In conclusion, we have realized synchronization and anti-synchronization simultaneously in the unified chaotic systems. To achieve this, a novel algorithm has been proposed to determine the variables of the master system that should anti-synchronize the corresponding variables of the slave system. Two different control strategies for the uniform chaotic systems has been presented to guarantee coexistence of synchronization and anti-synchronization. In all, numerical simulations have been employed to validate the proposed methods and to show the effectiveness of their. Finally, the method proposed in this paper can be used for other chaotic and hyperchaotic systems.

\section{References}

[1] L.M. PeCora and T.L. Carroll: Synchronization in chaotic systems. Physical Review Letters, 64 (1990), 821-824.

[2] S. Boccaletti, J. Kurths, G. Osipov and D. Valladares: The synchronization of chaotic systems. Physics Reports, 366 (2002), 1-101.

[3] S. BowOnG: Stability analysis for the synchronization of chaotic systems with different order: application to secure communications. Physics Letters A, 326 (2004), 102-113.

[4] D. HuANG: Simple adaptive-feedback controller for identical chaos synchronization. Physical Review E, 71 (2005), 037203.

[5] R. GuO and G. LI: Modification for collection of masterslave synchronized chaotic systems. Chaos, Solitons \& Fractals, 40 (2009), 453-457.

[6] R. GUO: A simple adaptive controller for chaos and hyperchaos synchronization. Physics Letters A, 372 (2008), 5593-5597.

[7] G. WANG: Stabilization and synchronization of genesiotesi system via single variable feedback controller. Physics Letters A, 374 (2010), 2831-2834.

[8] Y. ZHANG and J. SUN: Chaotic synchronization and anti-synchronization based on suitable separation. Physics Letters A, 330 (2004), 442-447.

[9] S.C.J. Hu and L. CHEN: Adaptive control for anti-synchronization of Chuas chaotic system. Physics Letters A, 339 (2005), 455-460.

[10] S. Hammami, M. Benrejeba, M. Fekib and P. Borne: Feedback control design for Rössler and Chen chaotic systems anti-synchronization. Physics Letters A, 374 (2010), 2835-2840. 
[11] M.M. Al-Sawalha, M. Noorani and M. Al-Dlalah: Adaptive antisynchronization of chaotic systems with fully unknown parameters. Computers and Mathematics with Applications, 59 (2010), 3234-3244.

[12] M. Srivastava, S. Ansari, S. Agrawal, S. Das and A. Leung: Antisynchronization between identical and non-identical fractional-order chaotic systems using active control method. Nonlinear Dynamics, 76(2), (2014), 905-914.

[13] H.-L. LI, Y.-L. JIANG and Z.-L. WANG: Anti-synchronization and intermittent anti-synchronization of two identical hyperchaotic chua systems via impulsive control. Nonlinear Dynamics, 79(2), (2015), 919-925.

[14] Q. ZHANG, J. LÜ and S. CHEN: Coexistence of anti-phase and complete synchronization in the generalized Lorenz system. Communications in Nonlinear Science and Numerical Simulations, 15 (2010), 3067-3072.

[15] R. GUO: Simultaneous synchronization and anti-synchronization of two identical new 4D chaotic systems, Chinese Physics Letters, 28 (2011), 040205.

[16] Z. WANG and X. SHI: Coexistence of anti-synchronization and complete synchronization of delay hyperchaotic Líu systems via partial variables, J. of Vibration and Control, 19 (2013), 2199-2210.

[17] S. KUNTANAPREEDA: Chaos synchronization of unified chaotic systems via LMI. Physics Letters A, 373 (2009), 2837-2840.

[18] Z.Q. ZHANG, H. SHEN and J.L. Li: Adaptive stabilization of uncertain unified chaotic systems with nonlinear input. Applied Mathematics and Computers, 218 (2011), 4260-4267.

[19] C.-J. Cheng and C.-B. Cheng: An asymmetric image cryptosystem based on the adaptive synchronization of an uncertain unified chaotic system and a cellular neural network. Communications in Nonlinear Science and Numerical Simulations, 18 (2013), 2825-2837.

[20] X.R. CHEN and C.X. LIU: Passive control on a unified chaotic system. Nonlinear Analysis: Real World Applications, 11 (2010), 683-687. 